\title{
PSYCHOLOGY
}

\section{A Theory of Anxiety Conditions}

\author{
Anonymous Author ${ }^{1}$
}

Correspondence

Email:

theory.of.anxiety.conditions@outlook.com

\begin{abstract}
This article is a summary of the notes I made whilst studying my own anxiety and my consciousness more broadly. The conclusions and theory I ultimately came to helped me a lot and so I have decided to share it on the off-chance it helps others. This article proposes that we may be able to identify the casual mechanism underlying a range of anxiety conditions by directly observing and formalising the components and dynamics of our conscious experience. However, as emphasised throughout, due to its nature this is only a theory about me, and can only ever be falsified on an individual basis. Therefore, I am keen to share the ideas and theory I came to in order to understand if others experience similar components and dynamics in their own conscious experience. If they do, I believe it could have significant implications for how we understand these conditions and the therapeutic approaches to treating them.
\end{abstract}

\section{1 | SCIENCE OF CONSCIOUSNESS}

Our consciousness is entirely unique when it comes to applying the scientific method. All other science is predicated on observing and explaining external reality using our consciousness. This means we have a real problem when coming to scientifically study consciousness itself, namely, we can't observe other peoples'. It also though has a unique benefit, we have a direct and intimate window onto our own. Therefore, when it comes to a conscious experience like anxiety, we are forced to make a choice for the scientific foundation of a theory.

On one hand, we can confine ourselves to only talking about the things we can observe in other people (symptoms, behaviours, biology etc.) so that we can make theories with empirical claims about all humans (or all people with anxiety). However, we then exclude the possibility of ever being able to observe a conscious causal mechanism that could be generating these empirical claims (if such a mechanism exists).

On the other hand, we can allow ourselves to talk about what we observe in our own consciousness so that we can potentially make theories which are directly based in conscious causal mechanisms. However, in doing so, we give up the ability to make empirical claims about other humans. These claims then can only ever be falsified on an individual basis. 
There are currently two main cognitive theories for anxiety conditions. There is Aaron T. Beck's 'General Cognitive Model'[1] and Albert Ellis's 'Rational-Emotive Behavior Therapy' (REBT)[2]. These theories are based in the first option for a science of consciousness and are designed to describe how we externally observe the symptoms and the mental processes of anxiety sufferers. They are inherently based in the idea that there is something 'irrational', 'dysfunctional' or a 'faulty informationprocessing system' going on in the sufferers mind. These theories have the benefit of being easily related to empirical evidence from studies of anxiety sufferers. The trade-off is that they cannot provide a causal mechanism that we can actually observe, because, from the perspective of these theories, we cannot observe what is actually going on in the sufferer's mind.

In contrast, the theory I will lay out in this essay is based in the second option. The theory will be developed by observing and describing the way I, and possibly you, directly experience our own consciousness and (if you have them) anxiety events. At their most basic these observations will be things like, "I experience thoughts popping into my head", or "I feel x, when y". The theory will suggest that we can identify the cause of anxiety conditions just through analysing these types of observations and thinking about the type of system they describe. It is also based in the idea that even severe anxiety does not need to involve any irrationality or dysfunction on the part of the sufferer.

Crucially, when it comes to evaluating the validity of this peculiar type of theory, you are the scientist, you are the only one who can observe what is going on inside your own conscious experience, and, as such, you are the only one who can ever decide whether the observations I make about myself, and the theory I will propose, is true for you.

Laying out this theory is going to be a journey with a number of steps.

First, I will try to show that when we study our own conscious experience the picture that emerges can be quite different to what we normally assume (or at least how I previously assumed), with dynamics I hadn't fully understood. In doing so we will formalise our conscious experience as a system which we can study.

Second, armed with this more formal description of our consciousness, we can describe how I/we experience anxiety events and conditions.

Third, I will identify a causal mechanism within the conscious system described that can explain how the phenomenon of anxiety conditions could arise. It will also explain why the experience of anxiety conditions has the general characteristics we observe.

Fourth, we will analyse the various factors that would in theory determine how this general causal mechanism would present itself in different contexts.

Fifth, I will show how this theory of anxiety conditions appears to match and explain the current diagnostic criteria for the main anxiety conditions described in the Diagnostic and Statistical Manual of Mental Disorders (DSM).

Finally, I will outline what this theory would imply for the treatment of anxiety conditions and how this relates to the current therapies.

\section{2 | STUDYING OUR CONSCIOUS EXPERIENCE}

The first step then is to study our own consciousness with the hope of formalising what we experience and its characteristics.

Before we begin, it is important to clarify what I mean by 'studying our conscious experience'. To be very clear, I don't mean 'studying our conscious experience' in an abstract way, I mean literally how would I describe to another human what it is like to be inside my head each day.

I realise to many people (including myself a few years ago) this question is likely to sound trivial to the point of not even needing to think about it. What's more, much of what I will describe in the next section I expect will sound very obvious and 
intuitive, such as, "I have thoughts that pop into my head". However, bear with me, as we will come to see, when we bring these seemingly trivial properties of our conscious experience together, the picture that emerges is of a system that is actually really quite complicated and quite different to how it is commonly viewed. Most importantly, it is this system which defines everything that is good, bad or indifferent about our existence, and so if there is anything that deserves a little extra scrutiny, it is what it means to have a conscious experience.

I now believe that for most of my life I missed a complicated and deeply important question hiding right behind my eyes. A question that has fairly profound implications for how I understand myself.

In practice, studying your own consciousness is about self observation and personal experimentation. It is about focusing not on the contents of one's conscious experience, but on the nature of the conscious experience itself. It involves thinking about things like: 'what are the properties of my thoughts?', 'what conscious experience am I describing when I say I have beliefs?', and 'what am I saying when I say I am experiencing a feeling?'. It may at times seem quite unusual and uncomfortable to look inwards like this at the events and processes going on inside your conscious experience but, as we will see, I believe we can learn a lot.

This essay then is fundamentally about what I observe when I look inwards at the properties and dynamics of my own conscious experience, and specifically how through these observations I came to understand what caused me to experience anxiety events. Given I can only ever study my own consciousness, I can only say that what I observe is true for me. However, as humans are fundamentally quite similar to each other, and from my discussions with others, I would wager there is a good chance the basic elements of my consciousness are pretty similar to yours. Therefore, to get the most out of this essay, I would highly recommend trying to see whether you can observe in yourself the concepts and ideas I express.

\section{I A MODEL OF MY CONSCIOUSNESS}

Now I have clarified what I mean by studying our conscious experience, I can now start to lay out how I came to see my own conscious experience when I studied it.

When I first looked inwards to study my consciousness, at any given moment it appeared much like a fuzzy mess of sights, sounds, ideas, sensations, memories, pains, predictions and many more. However, within this mess, there seemed to be common structures and relationships.

Many of these common structures and relationships are already heavily alluded to in how we currently discuss our conscious experience in common language. However, what we rarely seem to do, and what I will attempt to do here, is formalise these intuitions about our conscious experience into a set of distinct components that interact with one another. In doing so, we will develop a conceptual model of the system that seems to define our everyday conscious experience.

By analysing our conscious experience in this way, I believe we start to gain a far greater understanding of why our consciousness behaves in the way we experience. This way of describing our conscious experience will also allow me in future sections to formally analyse how and why phenomena like anxiety conditions could arise within this system.

\section{1 | The Components and Relationships within My Consciousness}

The first notable thing about our conscious experience is that it doesn't seem to be one homogeneous thing, instead there appear to be many components that have different characteristics. These components stand in relationship to each other, creating a system of interacting parts. Over time I came to see my consciousness as having four fairly distinct components, which combined capture all the fundamental characteristics of my conscious experience. These four components are as follows. 


\subsection{1 | Thoughts}

Thoughts refers to that conscious experience of having a transient flow of content (ideas, words, numbers, memories, predictions, concepts, observations, etc.) running through our mind, that seems to float somewhere between our ears. It would include conscious experiences such as thinking what to say, recalling memories, doing mental maths, making subjective evaluations of the world ('that was delicious'), having an 'inner monologue' and many more.

Very importantly though, thoughts seem to come in two discrete kinds:

First, there is the 'pop into your head' kind ("I forgot to turn the oven off", "crap that bus is about to hit me", etc.) which we seem to have no direct control over, I will refer to these as involuntary thoughts .

Second, there is the 'I'm choosing to do some thinking' kind (studying for exams, counting to 20, reading this essay, planning your holiday etc.), which we seem to have complete control over, I will refer to these as deliberate thoughts .

These two types of thoughts (involuntary and deliberate) seem to be in a kind of constant tug of war for air time based on which has the most important/useful information to convey at any one time. They also clearly affect one another, something will often pop into my head which then leads me to deliberately think about it or something related. Equally, deliberately thinking (for example about where to go on holiday) will along the way lead many memories and ideas to pop up into my consciousness. We can see this relationship between involuntary and deliberate thoughts at play in many common concepts such as "getting distracted", "trying to focus", "I suddenly remembered" and so on.

\subsection{2 | Feelings}

I will use feelings to refer to the conscious experience of sensory input (sight, sound, hot, shaking, tingling, taste, touch etc.). To be clear, when I say feelings I mean exclusively the conscious experience of sensory input, which we receive from our body (e.g heart racing, numb foot etc.) and the outside world (e.g sight, sound etc.). This definition then excludes evaluative judgments or interpretation of the sensory input, which would come under thoughts. For example, staring completely blankly out the window would be purely experiencing the feeling of sight. In contrast, the act of identifying objects or considering what was going on outside your window would be thinking about what the feeling of sight means or is telling us.

One way I can easily observe this key distinction between thoughts and feelings is to think about (remember) the last time I ate some chocolate. I might well remember what type of chocolate it was and how delicious it was. If it was recently I might even remember what I was thinking and doing at the time. However, sadly what I won't consciously experience when I remember eating chocolate is the actual sensation of tasting and smelling the chocolate (it would save me a lot of calories if I could). Here then we can neatly see the distinction, the conscious experience of remembering eating chocolate would be entirely thoughts (underpinned by beliefs), the conscious experience of the sensory input of taste and smell whilst actually eating chocolate would be the feelings.

Just as how we seem to have two types of thought that we have different levels of control over (deliberate and involuntary), the various feelings also appear to differ in how much influence we have. One crucial aspect of this is that different feelings seem to lie on a spectrum of how much power our thoughts have to bring them about and intensify them.

At one end of the spectrum we have basically exogenous feelings, these feelings can neither be generated nor meaningfully modified by our involuntary or deliberate thoughts. Good examples would include bright light, loud noise, someone tickling you and to a slightly lesser extent pain. In other words, our experience of these feelings are fairly independent of what we consciously think about, and crucially, experiencing these feelings is entirely contingent on some external event (i.e someone shining a torch in your eyes).

In the middle of the spectrum we find some partially endogenous feelings that can be meaningfully modified by our involuntary or deliberate thoughts but cannot be generated by them. A good example would be taste, if I sit in front of an engrossing Netflix program and idly eat a chunk of Toblerone I will often barely consciously experience the taste. In contrast, if I sat down 
in a quiet room and focused all my attention on the taste of the almond nougatey goodness and thought about how delicious it is as a slowly chewed it, I would consciously experience the taste much more intensely and I will likely consciously experience a number of flavours and textures that would of otherwise past me by. Hence, for a partially endogenous feeling like taste, my thoughts can have a meaningful impact on my experience of the feeling. However, they cannot generate them, sadly as noted, just thinking about chocolate is not sufficient to generate the conscious experience of tasting chocolate on its own, I still need the chocolate.

At the other end of spectrum we have some super interesting fully endogenous feelings that can be both generated and meaningfully modified by our involuntary or deliberate thoughts and so are really very intertwined with them. A good example being the group of feelings associated with anxiety and embarrassment (sweating, heart racing, shaking, 'feeling hot' etc.). There is clearly a direct causal link between me thinking and believing I am in danger and the feeling of sweating, because we know one of physiological symptoms of thinking you're in danger is, well, sweating. Therefore, my thinking about how bad it would be to really sweat through my top on this first date, does quite literally have the causal capacity to make me sweat through my top. Hence for these fully endogenous feelings our thoughts (based in our beliefs) can play a significant role in bringing them about and/or intensifying them.

This idea that there seems to be a subset of our feelings where our conscious experience of them can be meaningfully determined by how much we think about them (i.e focus on them), and what we believe about them, is an important observation and one that we will use throughout. Therefore, I will often lump partially and fully endogenous feelings together as just endogenous feelings.

\subsection{3 | Actions}

Actions are the expression of our conscious agency in the external world around us. This would include, how we decide to move, what we decide to say and so on. Actions are therefore the physical manifestation of a conscious choice and you have complete control over them (barring coercion or a lack of coordination).

Notably, one thing we exclude from actions is reflexes, which are never intermediated by our consciousness and therefore are not in our control. Reflexes would more properly be characterized as the physical equivalent of what we defined as involuntary thoughts.

\subsection{4 | Beliefs}

Beliefs are the most tricky component. Beliefs are not thoughts. Thoughts are inherently transient, we experience thoughts very directly, they are a flow, changing from one minute to the next. In contrast beliefs is a catchall for everything we currently take to be correct propositions about the world and ourselves (i.e beliefs encompasses what we might normally call our knowledge, convictions, religious beliefs, etc.).

Beliefs are built from many sources, from the essentially pre-programmed ("pain is bad"), to the mainly evidence based ("processed foods are unhealthy"), to the experience based ("there is a cafe round the corner"), to the purely subjective ("I like abstract paintings"), to the philosophical ("murder is always wrong"), to the largely faith based ("I believe in x God"), and so on. I also clearly hold different beliefs with varying degrees of certainty, from the so certain I don't even think about it ("this laptop exists outside of my own imagination"), through the fairly confident ("exercise is generally good for you"), to the if I had to guess I will assume its this but I m really not sure ("I wouldn't enjoy bungee jumping"). All these beliefs together form the lens through which we view ourselves and the world.

I find my conscious experience of beliefs quite strange, I could choose to deliberately have the thought "the earth is flat", I could deliberately remember the evidence given by flat earthers online, I could tell everyone "the earth is flat" and I could go to the flat earthers' conventions. However, even if I did all these things, I personally would still have this other conscious experience of "knowing" or rather believing that the earth is in fact not flat. I also seemingly can't directly control my beliefs, I can't just 
flip a switch and choose to believe the earth is flat (although to other people my actions might look like I had), the only way to "genuinely" change my conscious experience of the belief it seems would be to find reasons that I found compelling. Therefore, this conscious experience of beliefs (i.e holding things to be true) seems to be both very real but also very different to thoughts, feelings and actions.

One important subset of beliefs we will consider later are the beliefs we hold about ourselves. My beliefs about the capabilities of my body and mind inform how I try to achieve any task (e.g these beliefs mean I try to use my legs to run to the local shop rather than my arms to fly there).

Ultimately then beliefs provide the context in which our experiences of all the other components we have described occurs, beliefs influence what we think, what we do and how we interpret our feelings. Our beliefs though are clearly not set in stone but are also not fully under our control, they are constantly being updated by our experiences (i.e the combination of thoughts, feelings and actions).

\subsection{5 | (This isn't one!) Emergent Experiences}

The one aspect people might think is missing from the four components above are the overarching conscious experiences like love, happiness, awe, hatred, anxiety and so on (which are often colloquially described as feelings). The reason these have not been included is that they do not appear to be components in themselves. Instead to me they are better described as emergent properties of the conscious system containing the 4 components above. For example, I can't pin down a feeling or thought or belief or any other thing that is love, but my conscious experience of love definitely seems to involve feelings, thoughts and beliefs, it just seems to be a complex irreducible combination of all of them. Therefore, for our purposes, these aggregate / emergent experiences are not components in themselves but rather things we might seek to discuss / explain through the interactions and dynamics of the system containing the four components above.

\subsection{The Conscious System}

Combined these four components and their sub categories give us (I hope) a fairly ordinary description of what it is like to be conscious. My senses give me feelings that contain information about my body and the outside world, my involuntary and deliberate thoughts comprehend this information based on my beliefs about the world, and then through my deliberate thoughts I decide what actions to take. For me at least, and after much introspection, when I look inwards I can always split up what I am consciously experiencing in that moment into specific types of these general components.

We can also see from the definitions above that there are numerous ways in which these components interact with each other, some of the key relationships being: (1) deliberate thoughts often trigger related involuntary thoughts and vice versa, (2) deliberate thoughts can intensify (and in some cases generate) endogenous feelings, (3) our beliefs ultimately determine what we deliberately think about, our actions, and how we interpret our feelings, (4) our experience of the world (thoughts, feelings and actions) indirectly updates our beliefs, and (5) our actions will influence the situations we are in and in doing so the feelings and thoughts that are linked to our interaction with the external world.

If I accept this (I think) fairly mundane view that my conscious experience contains a set of different components which relate to one another, then I am also saying that I can study my conscious experience as a system, and a surprisingly complicated system at that. Given the number of components and the number of relationships between them, to lay out all the possible dynamics within this conscious system goes far beyond what I could cover here. Therefore, for this theory, I am going to constrain myself to only talking about the specific dynamics and relationships that are relevant for anxiety and so save the discussion for the next sections.

However, from the theory so far, some readers will have noted that the system I believe describes my consciousness is actually rather problematic. There is the possibility for feedback loops, in fact lots of them (e.g deliberate thought $->$ feelings - $>$ deliberate thought, involuntary thought $->$ deliberate thought $->$ involuntary thought, belief $->$ deliberate thought $->$ action $->$ belief). 
These feedback loops would be ok if I directly controlled all the parts of my consciousness. The problem is, I apparently don't. There are some parts I do directly control (actions and deliberate thoughts), some parts I have nearly no control over (involuntary thoughts and exogenous feelings) and others that I appear to only have some influence over (endogenous feelings and beliefs).

When it comes to my own consciousness then I appear to be in the same unenviable position as an engineer or banking regulator who finds the system they are trying to manage contains a feedback loop. The self reinforcing nature of feedback loops mean they can easily cause the system to spin out of control. If my description of the components above is broadly accurate then these feedback loops in our consciousness could exist, and as we will start to see, the malfunctioning of these conscious feedback loops can seemingly explain a lot.

Therefore, analysing the nature of this system that seems to make up our conscious experience appears to be both not trivial and also potentially quite important.

\subsection{Summary}

Overall, I hope we can see that by formalising some fairly basic observations about the different components of my conscious experience and their characteristics, we end up with a system that seems really very complicated. I also appear to have only partial control of this system (via deliberate thoughts and actions). What's more this system that makes up my conscious experience appears to be vulnerable to feedback loops. Therefore, this way of viewing my conscious experience suggests I was really quite wrong to assume I fully understood what my own conscious experience was. It suggests that in reality my conscious experience could easily have dynamics I would not necessarily expect.

At this point it makes sense to switch from considering my conscious experience in general, to considering how this model can be used to understand the cause and conscious experience of anxiety conditions. Everything we are going to discuss from here is going to be in terms of these components and the dynamics of the relationships between them. Therefore, if at this point you are thinking "no I don't experience thoughts popping into my head" or "no I don't experience/notice different feelings when a focus on different parts of my body / senses" or "no I can't deliberately try to think about something" (and so presumably don't experience free will?) or "no I don't have a set of beliefs that guide how I think and act" then it may not be worth continuing or it may be worth spending some time observing your own consciousness to see if you do start to see these components. However, if my bet is correct and you can also see in your consciousness broadly the same set of general components (thoughts, feelings, beliefs and actions), then I am going to switch in the next sections to talking about "our" consciousness rather than just talking about "my" consciousness.

Before we leave this section though, I think it is important to highlight how for me this view of our conscious system also significantly changed how I saw myself. It suggested that maybe 'I' am not really a conscious mind, atop a body sitting in the external world. Maybe 'I' am actually just a small agent, sitting in a corner of conscious mind, atop a body sitting in the external world. As this agent, I have some direct control that I can exert within this internal conscious world (through deliberate thoughts and, to a lesser degree, actions), but the system that describes this conscious world is much larger and more complicated, with many parts that ' $\mathrm{I}$ ' as the agent can at best only indirectly influence (feelings, beliefs, involuntary thoughts and all my subconscious). Seen this way I started to recognise that many conscious experiences I previously thought of as being very much part of 'me' (involuntary thoughts, feelings, beliefs etc.) were actually in many ways more similar to the external world in practice. This perspective that ' $I$ ' am not everything that is in my head but rather a small part of my consciousness (that which I can control) looking out and interacting with a complex inner world outside 'myself' (that which I can't control but can only influence) fundamentally changed how I viewed myself. It raises what I think is a vastly important question - how should 'I' choose to behave within this complex inner world I find myself trapped in? We spend decades learning how we should use our actions to successfully interact with the external world but seemingly no time at all trying to understand how we should use our deliberate thoughts to successfully navigate and comprehend this complex inner world. 


\section{4 | ANXIETY CONDITIONS}

Now we have a model of our conscious experience, the next step towards developing a theory of anxiety conditions, is to pin down what we mean by anxiety conditions and the conscious experience they describe.

\section{1 | Theoretically - Anxiety as a Separate Condition}

All humans get anxious, it's completely normal. Therefore, when we talk about anxiety as a condition in itself, we are talking about the condition where the sufferer recognizes their mental and physiological anxiety response to a situation is disproportionate to how they believe they should respond to that situation. In other words, where the disproportionate nature of the anxiety response itself is the object of study. Whenever I say anxiety in this essay, I mean anxiety as a separate condition.

\section{2 | Practically - The Conscious Experience of Anxiety}

I am only writing this because I experienced getting anxiety, being diagnosed with anxiety and having cognitive behavioural therapy. Unless you have observed a conscious experience yourself it is really very hard to comprehend what is actually happening in someone else's consciousness when they say they have it. The best we can do is get that person to describe what they experience going on in their head as objectively as possible. Therefore, I wish to briefly outline how I experienced anxiety, using the four components of my consciousness I defined in the previous section.

In the past I have experienced a number of the different 'types' of anxiety as medicine currently defines. The process I describe below is intended to be a general description of how I observed an anxiety event (aka attack) in myself. Across all 'types' of anxiety there was some form of the first three phases below, because it is these phases of an event that involves experiencing the disproportionate response which defines anxiety. Whether the other phases occurred really depended on the nature of the fear the particular 'type' of anxiety related to.

\subsection{1 | Event - Phase 1 - Trigger}

An anxiety event starts with what might be an involuntary thought (oh no what if?, I forgot to go to the toilet), an external event (going to a confined space) or a feeling (I have butterflies / my hand is tingling / I am sweating). It is often a concoction of the three in quick succession. The moment of the trigger would never be that bad in itself but rather the small rock that sometimes starts the avalanche. However, once you get used to a particular anxiety, you obviously learn either explicitly or subconsciously the logic of this mental process and realize what a trigger leads to.

\subsection{2 | Event - Phase 2 - Focus}

Once the trigger has brought the thought of something you fear happening (and so believe is important) into your consciousness you start to think (deliberately and often involuntarily) about whether you are experiencing the feelings you have associated with that fear occurring, am I sweating / do I feel like I need the toilet / why are my hands tingling etc. In other words, you start thinking intensely about what your current conscious experience means about the probability of the fear happening. 


\subsection{3 | Event - Phase 3 - Escalation}

The focus on the feelings associated with something you fear triggers more negative thoughts (oh no I don't want to embarrass myself on a date by sweating, I can't mess up this presentation, I am stuck on this tube and need the toilet), this of course makes you focus more on whether the feeling is still there and crucially if it is getting worse. The negative feelings appear to escalate and negative feelings bring more negative thoughts and with every cycle the fear seems to become increasingly imminent (why is this happening, what is the worst case scenario, what if $\mathrm{x}$, what does this mean). Thoughts beget feelings and vice versa as you tumble down into an increasingly intense state of anxiety. It feels to me almost like this process sucks more and more of your conscious attention inwards, dragging you into utterly focusing on this apparently increasing danger.

\subsection{4 | Event - Phase 4 - Call to Action}

The call to action only really seems to occur in certain types of anxiety where your evaluation of the probability of the fear occurring can get near $100 \%$. As you become ever more stressed both due to the feelings being amplified, shaking, sweating etc and your evaluation of probability of the fear becomes increasingly high (your heart drops, aaaaah, its happening now), the option of escape naturally pops into your head. Jump off the tube, run to the bathroom, leave the party, whatever. It seems almost irresistible and for good reason, this is a logical out from something you do believe might be really bad, and even more importantly, do believe is very likely. Yet I can utterly promise you that the call to action is just a thought, it has no actual control over your actions. It has huge power to influence your calculation of what to do but there is not a direct causal link, it is intermediated by your deliberate thoughts. The question is whether you follow that intense desire to escape. If you do, this is likely the end of the anxiety event, but not the end of the anxiety. We will come to what if you don't much later.

\subsection{5 | Post Event - Phase 5 - The Analysis}

If the level of anxiety reached in the event is severe enough, the occurrence of events themselves will likely become something you are (completely understandably) anxious about. This gives rise to the endless hours spent thinking about why the event happens, what the worst case scenarios are, how bad it was or would be, trying to rationalize yourself out of it, work out the best strategies, etc. The thinking is not physiologically that unpleasant in itself, you may feel stressed or uneasy but nothing like the event.

\subsection{6 | Post Event - Phase 6 - Behaviour Change}

On the basis of the Analysis, and in the absence of changing the beliefs that led you to the event above, the rational conclusion is to consider avoiding situations where something unpleasant seems to always/often happen. Decide not to get the tube, go to the theatre, see friends, whatever. This is obviously not ideal but it is a trade off that has a perverse logic. This process will also make the event that much more inevitable if you are forced by virtue of work or luck into the situation you have been deliberately trying to avoid.

\section{3 | Summary}

These two descriptions of anxiety conditions layout what we are seeking to explain with the following theory. In the abstract, the theory will seek to explain the phenomenon of people who view their own excessive anxiety response as an issue in itself that negatively affects their lives. In practice, the theory will seek to show how the general conscious experience of anxiety events described above can be explained purely via the dynamics of the conscious system we have described. 


\section{5 | A THEORY OF ANXIETY}

Now we know what we are hoping to explain and have described a model of our consciousness we can use to discuss it, we can now turn to the theory I wish to propose. Before I do though, there is one insight that is utterly fundamental to everything we are about to discuss and so I wish to state it up front.

Most people have (unsurprisingly) not gone through the process of analysing and formalising their conscious mind and its capabilities (in a manner similar to what we did in the section 'A Model of My Consciousness'). We also get no formal education on what to expect from our conscious mind. Therefore, people may well hold mistaken beliefs about the nature of their consciousness, such as, which parts of their consciousness they control, what feelings mean and what we can or should try to do about the feelings or involuntary thoughts we experience.

As we will come to see, in exactly the same way a human who believed they could use their arms to fly would rationally throw themselves out a window leading to physical injury, humans who hold incorrect beliefs about how their consciousness works could rationally decide they should or must do/think things that lead to very negative states of mind. As this theory will contest, I truly believe it is possible that many people with anxiety conditions are not in any way irrational or biologically different, they may just be acting on a set of incorrect beliefs about their own consciousness. I think this possibility should be deeply concerning for all involved, as it means there is a chance we are medicalising something that may be caused by a lack of understanding, which would be more appropriately dealt with via the mental equivalent of sexual education.

The next section will lay out a general conscious process, which combined with the idea that we may hold mistaken beliefs about our own consciousness, can explain the general characteristics observed in anxiety conditions. We will then formalise the factors that determine how this conscious process would present itself in different contexts and then show how the theory so far can explain the various characteristics of specific anxiety conditions as clinically diagnosed.

I realise these are highly significant claims and I may well be wrong if my personal experience has been atypical. Therefore, before we continue I wish to emphasise once again that this is purely a theory and a theory derived entirely from analysing my own conscious experience. It is clearly a theory that I believe to be true of myself. The reader can also through introspection consider whether they think it might be true of themselves.

\subsection{Anxiety as a Conscious Feedback Loop}

\subsection{1 | The Feedback Loop}

When I identified the components of my consciousness I noted there appears to be a subset of feelings where our conscious experience of them can be generated and/or intensified by our thoughts, we called these endogenous feelings. We also noted how our beliefs about the world and ourselves ultimately determine what we decide to do and what we chose to deliberately think about.

If we accept then that we have feelings that get amplified (and sometimes brought into existence) based on how much we consciously think about them, and if we hold beliefs that lead us to see changes in these endogenous feelings as important (i.e maybe we believe it would be bad to sweat and embarrass ourselves or maybe we believe tightness in our chest might suggest a heart attack etc.), then we can start to see there is clearly the possibility of a feedback loop in our consciousness.

We experience an endogenous feeling, our beliefs lead us to think this feeling could be important, so we focus our deliberate thoughts on the feeling, this focus amplifies our experience of the feeling, this leads us to re-evaluate how important the feeling is and so we focus even more on the feeling and what it might mean, this amplifies our experience of it further, and so on. 
Involuntary thoughts will often play a secondary role in this feedback loop. An involuntary thought may well be what starts the feedback loop by bringing the endogenous feeling to our attention. We also discussed how deliberately thinking about something will often lead to related involuntary thoughts. Therefore, as the feedback loop iterates, we are also likely to consciously experience lots of thoughts popping into our head that are related to why we believe the endogenous feeling could be important.

This feedback loop appears to be a completely normal part of a human's consciousness, if we agree we have thoughts and we agree that what we focus on with our deliberate thoughts can affect how we experience certain feelings, then the possibility of this feedback loop is very real and a part of everyone of us.

However, the possibility of this feedback loop has a really very important implication. It suggests that seemingly extreme or abnormal states of mind could come about without any biological defect or inherent cognitive dysfunction. They could occur simply through an otherwise 'normal' person getting caught in a feedback loop between the components of their consciousness, due to the person being in a situation where they believe one of their endogenous feelings is related to something important. In such a situation the person would respond entirely rationally (under their beliefs), but their rational responses would sustain an escalating process of intensifying endogenous feelings and increasing focus that ultimately results in a state of mind which could appear both extreme and abnormal. As I will turn to next, this possibility can seemingly explain what causes anxiety events and many of the unique features we observe in anxiety conditions.

I also wish to recognise that, unless you are someone who has regularly experienced it, the possibility of this type of conscious feedback loop is likely to be hard to accept. The reason for this is the same as the reason people find it very hard to believe the banking system could collapse in few days, until they watch it happen. When a system (financial or conscious) is relatively stable under most conditions, and most of the time changes are small and largely linear, the idea that under specific conditions large non-linear changes could occur simply due to the situation facilitating a feedback loop does not fit with our natural intuition. Yet unless I am fundamentally wrong in the way I described the basic components of our conscious mind, it seems evident this feedback loop is possible, and, as such, its potential to generate extreme or abnormal outcomes could be very important. Note also that so far our discussion of this feedback loop doesn't actually mention a fear or anxiety. It is far broader and I believe could be important in many other areas of science related to human consciousness.

\subsection{2 | The Anxiety Feedback Loop}

We only need to add one condition to our description of the feedback loop above to make it about anxiety. This is that the beliefs that lead the person to think their endogenous feelings are important, are specifically beliefs that these endogenous feelings provide information about the probability or severity of something the person fears.

The anxiety feedback loop would now be as follows. The person experiences an endogenous feeling, they believe this feeling provides information about the probability / severity of something they fear, so they focus their deliberate thoughts on the feeling, this focus amplifies their conscious experience of the feeling, this leads them increase their evaluation of the probability / severity of something they fear, and so they focus even more of their attention onto the feeling and the possibility of the fear, this amplifies their conscious experience of the feeling further, and so on. Throughout this process as the person repeatedly increases their evaluation of the probability / severity of something they fear happening (based on their observation of escalating endogenous feelings) they will entirely rationally become increasingly anxious and experience the associated symptoms of anxiety.

I also think the experience of this anxiety feedback loop should actually be quite recognisable to many people, even those who do not have anxiety as a condition. Therefore, it is worth taking a moment to provide a detailed example, I think one particularly common experience would be as follows.

You are going to give a presentation (or do some other public speaking), in the days leading up to the presentation you are a bit anxious about it. When you get in the lift to the meeting holding your presentation notes, you notice that you are a bit sweaty and your hands are shaking a little (i.e an involuntary thought about things you are feeling). If you have given lots of presentations then you probably now largely brush off these feelings and consider them completely 'normal' and go back to planning what to say. However, if this is your first presentation or a particularly important presentation then you might well think something along the lines of 'I really hope no one notices my hands are shaking / I m sweating, that would be embarrassing'. This thought, 
based in the belief that shaking and sweating (endogenous feelings) are related to embarrassing yourself (a fear), leads you quite rationally to focus on how much you are sweating/shaking and how noticeable this might be. As you focus on the sweat on your brow or the slight shake of the piece of paper in your hand, you become more aware of them and consciously experience them more intensely. Experiencing them more intensely leads you to increase a bit your assessment of how likely you are to embarrass yourself, 'I hope I don't sweat through my top' or 'I should really calm down'. However, as shaking and sweating can be caused by thinking a fear is present (i.e they are endogenous), the fact you now believe embarrassing yourself has become more likely and are thinking more about the possibility of embarrassing yourself, means you will likely start sweating and shaking a little bit more. As you observe feeling hotter and shaking slightly more, you may well start to think something along the lines of 'oh no its getting worse', 'could I hide my sweat patches', 'how can I stop this happening' and so on. In a bitter irony, these thoughts will lead you to focus ever more attention on the seeming increasing probability of embarrassing yourself and what it means / what you can do, thus begetting another increase in sweating and shaking and the spiral continues. As you walk into the room you may well now feel pretty intensely anxious, despite being fairly calm when stepping into the lift. However, if you go ahead with the presentation, your focus (deliberate thoughts) is usually forced to switch away from your feelings and onto discussing the slides and remembering what to say, and so the feedback loop is broken and the symptoms of anxiety will gradually fade back to normal.

This is only a description of one way, one particular type of anxiety could play out in one particular situation, but I hope it serves to illustrate how the feedback loop I describe is not just an abstract theory but a causal description of what happens in our consciousness when we experience anxiety events in common everyday situations.

This anxiety feedback loop then appears to provide both a theoretical explanation for what could cause a 'normal' human to become apparently excessively anxious in specific situations, and a practical description of what it is like to experience an anxiety event.

It's also important then to recognise that lots of people who are never diagnosed with clinical anxiety do seem to experience instances of this anxiety feedback loop throughout their lives (with public speaking, exams, interviews, first dates, being some of the most culturally accepted). Therefore, it seems an accepted part of life that even 'normal' humans at times get excessively anxious. Under this theory these instances can be seen to involve and be explained by fundamentally the same conscious process as those cases we diagnose as clinical anxiety, the only difference is in degree and persistence.

\subsection{3 | The Anxiety Feedback Loop and the Characteristics of Anxiety Events}

The dynamics of this anxiety feedback loop would also appear to fit many of the notable characteristics of anxiety events, including those we discussed in the section 'Anxiety Conditions', namely that:

- Anxiety events have a trigger - this can now be seen as the thing (event, thought or feeling) that leads you to initially focus on the endogenous feeling you believe is related to the fear, and in doing so starts the feedback loop.

- Anxiety escalates over time without notable changes in the external world - the dynamics of the feedback loop explains how the interaction between thoughts and endogenous feelings can lead our conscious experience to escalate without any notable change in the outside world.

- Anxiety events are a conscious phenomena i.e the symptoms don't just happen without us knowing and we feel part of the process - under this theory the feedback loop is sustained by our own deliberate thoughts and beliefs, and so for the feedback loop to occur it must involve us being consciously aware of it.

- Anxiety events lead to an anxiety response that is disproportionate to what the external situation would suggest is reasonable - the feedback loop explains how through successive rounds of re-evaluation of the probability of the fear (based on escalating endogenous feelings) the person can become far more anxious than even their own initial evaluation of the situation would suggest was appropriate. 
However, a key observation that this anxiety feedback loop cannot yet explain is that anxiety conditions present themselves as being highly context specific and clearly not everyone is diagnosed with an anxiety condition. In order to explain this we should now consider what conditions need to be in place for this anxiety feedback loop to actually occur.

\subsubsection{Two Necessary Conditions for the Anxiety Feedback Loop}

If we look back at our description of the anxiety feedback loop we can identify that there needs to be two necessary conditions in place for the feedback loop to be possible.

First Necessary Condition: The person must be in a situation where they believe their endogenous feelings are meaningfully related to the probability of some fear occurring. Without such a belief they would not have a reason to focus any more of their attention onto the endogenous feeling and so the cycle would never get started or be sustained.

This is why anxiety often appears as being very context specific, in most contexts people don't believe the subset of feelings that are endogenous provide meaningful information about something they fear. For example, in most of life's situations (home, car, family, sitting at your desk etc.) people don't believe sweating and appearing anxious is much of a big deal, on first dates and in important presentations though people often believe it is a big deal, hence the feedback loop would be context dependent. It also explains why anxiety is most commonly observed in everyday situations where it is easy to see how someone can believe endogenous feelings are related to the fear e.g social situations, confined spaces and personal health. In contrast, anxiety conditions about, losing a loved one, losing your job, nuclear war, crime, natural disasters etc., which are often much more severe fears, but which are far harder to believe are related to our own endogenous feelings, are much less common.

Second Necessary Condition: The person must not fully understand the dynamics of this feedback loop in their consciousness. If the person did fully understand (even intuitively) that the act of focusing on the endogenous feeling is what is causing it to intensify, then changes in the endogenous feeling would not provide a reason to focus ever more attention onto whether the fear is becoming more likely. Hence they would not focus much additional attention on the feeling and as such it would not continue to escalate (or would escalate only very slowly).

Here we see the potential importance of the insight that humans may hold incorrect beliefs about the nature and dynamics of their conscious mind (I certainly did). In my experience it is this second condition that can sometimes make anxiety seem so confusing and upsetting to the sufferer, the anxiety feedback loop often feels like something you 'should' be able to control or as if you are somehow 'doing it to yourself'.

Therefore, we can see that whilst under this theory everyone of us sadly contains the potential for the anxiety feedback loop, even this basic feedback loop requires two fairly specific conditions for the escalating process we see as an anxiety event to be possible. This is why most people do not experience anxiety as a separate condition and why anxiety is observed as being very context specific. I will discuss the nature of these beliefs and the contexts in which they occur in much more detail later on.

\subsection{5 | Summary}

Overall, hopefully we can see that the theory that anxiety events are caused by a feedback loop between our deliberate thoughts and our endogenous feelings could explain many of the general characteristics of anxiety events that we observe in reality, and more importantly, it fits with how we observe anxiety events playing out in our own consciousness.

We can summarise the theory so far via a new definition for anxiety conditions:

Anxiety as a separate condition can occur when an individual believes their endogenous feelings are related to something the individual fears. This belief, combined with incorrect beliefs about the dynamics of their own conscious mind, creates the possibility for erroneous feedback loops within the individual's consciousness, leading to the experiences associated with anxiety. 
From this definition we see our theory suggests anxiety conditions could occur in any human. However, for it to be possible in practice there must be two necessary conditions, (1) the person believes their endogenous feelings are related to something the individual fears, and (2) the person does not fully understand that the escalating feeling could be caused endogenously. If these two conditions are in place and the person finds themselves in the situation where they believe the fear is possible, then a trigger is all that is needed for an anxiety event to occur.

It is important to note though that there may be biological, social, and cultural factors that predispose an individual to holding these beliefs. For example, women are often more scrutinised on their appearance than men, and so it is conceivable that more women might believe an endogenous feeling such as sweating is related to the fear of embarrassment in social situations. Equally, it is conceivable that someone who is generally more risk averse would be more likely to consider whether their endogenous feelings might provide information about the risks they wish to avoid, and in turn make it more likely they establish beliefs that would cause the anxiety feedback loop. However, whilst these factors may well affect the incidence of anxiety conditions we observe in the population, under this theory they are not the cause of the condition itself.

Finally, I wish to emphasise how this theory suggests people who have anxiety conditions may not have anything biologically or medically "wrong" with them. Instead it suggests anxiety may be the result of people holding completely understandable mistaken beliefs about the dynamics of their consciousness. If true this clearly has significant implications for how we should discuss and treat anxiety, which we will come to later.

At this point we have largely completed the core tenants of a theory of anxiety. We have identified the key components of our conscious experience, explained how a system containing these components would contain feedback loops, and identified a feedback loop that fits with and would explain many of the core observations of anxiety events.

\subsection{Factors Affecting the Anxiety Feedback Loop}

The next step is to dig deeper into the dynamics of this anxiety feedback loop and the factors that determine its intensity, duration, presentation and characteristics. From our discussion so far we can see three general factors we have touched on that would influence the dynamics of the feedback loop.

\subsection{1 | Factor 1 - The belief about how closely endogenous feelings are related to the fear}

We discussed previously how for this feedback loop to occur the individual must hold a belief that their endogenous feelings are related in some way to the severity or probability of something they fear. The fact this belief must exist for the anxiety feedback loop to occur gives rise to our first factor for determining the feedback loop's characteristics. This factor is how close the person believes this relationship is.

We can think of this belief as lying on a spectrum. At one end, the person believes endogenous feelings are only one of many factors that cause (or are evidence of) the fear, I will refer to this as a weak relationship. At the other end, the person believes the endogenous feelings are the only factor that causes (or are all the possible evidence of) the fear, I will refer to this as a strong relationship. Where the person's belief lies on this spectrum will determine two characteristics of the feedback loop.

First, the belief about how close the relationship is will partly determine the intensity of anxiety events. If the person only believes in a weak relationship then escalating endogenous feelings on their own will not lead the person to think the fear is very likely. Hence, a person with this type of belief about the relationship will likely experience only relatively mild anxiety events (or not even consider them anxiety events).

In contrast, if the person believes in a strong relationship then the person will believe the fear is very likely just on the basis of escalating endogenous feelings. Hence, someone who believes in a strong relationship is likely to experience more intense anxiety events. 
Second, the belief about how close the relationship is will partly determine the duration of anxiety events. If the person believes in a strong relationship then we would expect to see much more time limited anxiety events. This is because if you believe $\mathrm{x}$ directly causes (or is all the evidence for) $\mathrm{y}$, once you observe $\mathrm{x}$ (escalating endogenous feelings) then either $\mathrm{y}$ (the fear) happens fairly soon and your belief is correct or for some reason it doesn't (maybe you leave the situation or maybe your belief turns out to be incorrect).

In contrast, if the person believes in a weak relationship then anxiety events can be much longer and persistent. This is because if you believe $\mathrm{v}, \mathrm{w}$ and $\mathrm{x}$ cause (or is all the evidence for) $\mathrm{y}$ and you observe only $\mathrm{x}$ (i.e escalating endogenous feelings) then your belief that $y$ (the fear) might occur is not falsified simply by letting the anxiety event play out. This could lead to much more drawn out anxiety events of persistent worry where the possibility of the fear is simply seen as more likely but is neither confirmed nor falsified.

Overall then, we would expect that where the person's belief about the relationship sits on the spectrum from weak to strong is a key factor in determining the intensity and duration of the anxiety feedback loop. As a rule of thumb, if we are talking about partially endogenous feelings then we are more likely to observe a belief in a weaker relationship, whereas fully endogenous feelings are more likely to be associated with a belief in a strong relationship.

\subsection{2 | Factor 2 - Beliefs about how bad the fear would be}

Our theory is consistent with the idea that the individual's experience of anxiety is entirely rational based on their personal set of beliefs. Therefore, this is quite a simple one. If you believe the fear happening wouldn't really matter, you will never have an anxiety event because you won't be bothered to spend the time deliberately thinking about if it might be happening (and so the feedback loop never starts). If you believe the fear is quite bad (e.g for me, embarrassing myself) you could get anxiety events that escalate to fairly intense anxiety but not extreme. If you believe the fear is very bad (often death) then you could get anxiety events that escalate to very intense anxiety / panic.

Note that I said 'could' a lot, clearly there are two factors at play. One is how bad the person believes the fear would be, the other is how likely the person believes the fear is based on the escalation of endogenous feelings (i.e the first factor we discussed above).

Therefore, we can now see that the dynamics of the feedback loop are such that the intensity of anxiety events should be determined by a combination of how bad the person believes the fear is (factor 2) and how likely the person believes the fear is based on only their observation of the escalating endogenous feelings (factor 1). This should allow us later to make some interesting observations about whether the incidence of anxiety we observe in reality reflects this implication of the theory.

\subsection{3 | Factor 3 - Beliefs about the trigger}

When someone experiences anxiety events, they will likely find the fact they appear to get disproportionately anxious quite unusual and concerning. This will often lead them to develop beliefs about why this could be and in what situations it could happen. These beliefs may be entirely accurate. However, what is of interest to us, is that the person may hold incorrect beliefs about when and where they can experience this anxiety feedback loop. In other words, they may hold incorrect beliefs about what triggers their anxiety and why.

The reason this could be so important is that the first necessary condition for the anxiety feedback loop refers to the person "believing" they are in a situation where their endogenous feelings are related to the fear. Hence, it is possible the person believes they are in such a situation when in fact they are not, or unfortunately more commonly, the person actually is in such a situation but they do not believe it can happen.

To give a personal example, the first time I experienced the anxiety feedback loop was on a crowded train. Even though I had taken the train thousands of times before, I developed the belief that the possibility of getting very anxious required me to be on the train. As such I never experienced anxiety events on buses, in lifts etc, because I never thought it possible and so never 
focused on my endogenous feelings and so never triggered the feedback loop. Then a month or so later I sat down in a crowded theatre and an involuntary thought struck me, "this is a bit like the train, I hope I don't have an anxiety event", needless to say this idea that maybe an anxiety event was possible in this situation as well meant I focused on my endogenous feelings and in doing so triggered the anxiety feedback loop.

Therefore, we can see how the person's beliefs about which situations or events can trigger the anxiety feedback loop can significantly influence the specific presentation of a person's anxiety. We can also see the potential for shedding some light on the phenomenon of someone's anxiety "spreading" or becoming more generalised.

\subsection{4 | Summary}

These three factors then allow us to start to explain why the general anxiety feedback loop we described in the previous section could present itself in numerous different ways.

Now we have formalised the components of our conscious mind, outlined a feedback loop that could describe the general experience of anxiety, and discussed the main factors that would determine how this general feedback loop should present itself, we can turn to seeing if this theory could explain the presentations of anxiety as clinically observed.

\section{6 | EXPLAINING THE DSM}

In order to see if this theory could be an accurate description of reality, I will now try to show how it can describe the range of anxiety conditions as defined in the Diagnostic and Statistical Manual of Mental Disorders (DSM) [3]. I will exclude the two anxiety conditions that most commonly occur in children, Selective Mutism and Separation Anxiety Disorder, as I have no recollection of what it was like to be a child and so would not want to comment on if this theory is plausible. I will also exclude those anxiety conditions in the DSM which relate to substance abuse or other medical conditions.

In order to be comfortable that our theory is a possible explanation for a DSM condition we will need to show two things. First, that the DSM diagnostic criteria for the condition suggests that the two necessary conditions for the anxiety feedback loop are fulfilled. Second, that we can see how plausible versions of the three factors above could explain why the anxiety feedback loop presents itself with the particular characteristics described in the DSM.

For this discussion of the DSM though it appears the second necessary condition for the anxiety feedback loop to occur will always hold by definition. This is due to the DSM definitions not containing any reference to the primary cause of any of the conditions being a conscious feedback loop between deliberate thoughts and endogenous feelings. Therefore, we must assume that if someone is diagnosed with a condition as defined in the DSM, the dynamics of the feedback loop in the person's conscious experience are not fully understood. Therefore, to avoid repetition we will take the second condition as given.

Before we begin I would like to emphasise that there are lots of very common fears, that if they were also common in the DSM descriptions of anxiety conditions, would invalidate this theory. Any anxiety condition in the DSM which was defined by fears that are not easily related to our endogenous feelings / involuntary thoughts would suggest our theory was wrong, as the feedback loop could not occur. Therefore, we are relying on the DSM largely excluding huge swaths of things humans often worry about, from the extreme (war, natural disasters, crime) to the common (headaches, broken bones, losing your job, losing a loved one, break ups). In fact, we are excluding so many common human fears, them not appearing in the DSM description of anxiety conditions would be notable in itself. 


\section{1 | Social Anxiety Disorder ([3], p.202-208)}

The DSM diagnostic criteria for Social Anxiety Disorder covers experiencing "out of proportion" anxiety in a range of situations that involve social interactions from "having a conversation" to "giving a speech" and numerous others. It covers many possible fears, including that the person will be "judged as anxious, weak, crazy, stupid, boring, intimidating, dirty, or unlikable". Most importantly, the DSM notes that:

"The individual fears that he or she will act or appear in a certain way or show anxiety symptoms, such as blushing, trembling, sweating, stumbling over one's words, or staring, that will be negatively evaluated by others"

The reader will note that these largely fall into the category of endogenous feelings or involuntary thoughts. "Blushing, trembling, sweating, stumbling over one's words" are all symptoms of either thinking you are in danger or thinking you are embarrassing yourself, i.e they are endogenous. "Staring" it seems is describing an involuntary thought, on first dates and in one on one meetings it seems common for the thought of "oh no, am I looking at them weirdly" or "am I staring at them" to pop into your head (or at least my head), thus triggering the anxiety feedback loop.

Crucially these endogenous feelings / involuntary thoughts are related to a fear i.e being "negatively evaluated by others". Hopefully then it is fairly evident that the DSM diagnostic criteria for Social Anxiety would fulfill the first necessary condition we identified for the anxiety feedback loop to occur, namely, that the person must be in a situation where they believe their endogenous feelings are meaningfully related to the probability of some fear occurring.

For a more detailed description of the feedback loop involved in Social Anxiety it is worth going back to the description we gave of giving a presentation in the section 'The Anxiety Feedback Loop', which we can now see would fall under the DSM definition of Social Anxiety.

Unfortunately the DSM definition of Social Anxiety Disorder contains such a broad range of fears, situations, and outcomes (including "panic attack") that it is hard to relate it to many specific versions of the three factors affecting the feedback loop's dynamics, as all are possible.

Overall though, Social Anxiety Disorder appears to be a fairly textbook case of an anxiety condition our theory would suggest. Being negatively evaluated by others is a fear that is clearly very easily related to the endogenous feelings associated with anxiety and embarrassment, hence the first condition holds. The second necessary condition holds by definition and social situations represent a clear and common trigger for the feedback loop to start. The way a social anxiety event progresses also appears to fit with the description of a feedback loop.

\section{2 | Panic Disorder ([3], p.208-214)}

Panic Disorder is defined in the DSM as "recurrent unexpected panic attacks" where a panic attack is taken to be "an abrupt surge of intense fear or intense discomfort that reaches a peak within minutes". Crucially, panic attacks specifically must contain four of the following 13 symptoms.

- "Palpitations, pounding heart, or accelerated heart rate."

- "Sweating."

• "Trembling or shaking."

- "Sensations of shortness of breath or smothering."

• "Feelings of choking."

- "Chest pain or discomfort." 
- "Nausea or abdominal distress."

- "Feeling dizzy, unsteady, light-headed, or faint."

- "Chills or heat sensations."

- "Paresthesias (numbness or tingling sensations)."

- "Derealization (feelings of unreality) or depersonalization (being detached from" one- self).

- "Fear of losing control or "going crazy.""

- "Fear of dying."

Once again, these symptoms that define panic attacks are largely feelings that could be endogenous. In fact for the DSM they are by definition endogenous feelings as another criteria for Panic Disorder is that "the disturbance is not attributable to the physiological effects of a substance (e.g., a drug of abuse, a medication) or another medical condition (e.g., hyperthyroidism, cardiopulmonary disorders)", i.e they must be generated endogenously.

Most interesting though are the two final "symptoms", namely, the fear of losing control, going crazy or dying. Therefore, in Panic Disorder, we have a range of feelings that can occur when a human believes they are in extreme danger, and these endogenous feelings are often related to the fear of them losing control, going crazy or dying. Therefore, the DSM diagnostic criteria for Panic Disorder also fulfills the first necessary condition for the anxiety feedback loop.

Unlike Social Anxiety the DSM definition of Panic Disorder is actually quite specific and so the factors we discussed should help explain its unique presentation, i.e very sudden and very intense anxiety events.

First, this is clearly a strong relationship (factor 1) as the person (in this case incorrectly) believes the endogenous feelings that occur in a panic attack (heart racing, sweating, shaking etc.) are evidence of the fear, and crucially it is evidence the fear is happening right now (i.e it is all the evidence you require to believe the fear is happening).

Second, the fear in this case is often about as severe as possible (factor 2), namely, loss of self or loss of life.

These two factors combined mean our theory would suggest that the situation described in the DSM diagnostic criteria would generate an anxiety feedback loop that could lead the person to entirely rationally (under their beliefs) become nearly as anxious as it possible for a human to be. Hence, with this theory and the specific beliefs/situations described in DSM, the fact the person panics can be seen as entirely expected.

There is something in this description though that might trouble the reader. We are essentially saying that if you believe the endogenous feelings involved in panic attacks are really bad (because you believe they are evidence you are about to experience loss of self or loss of life), then this belief is all you need to enable the anxiety feedback loop to escalate your endogenous feelings to the point of a panic attack.

In other words, our theory would suggest Panic Disorder does not need any external situation to occur (unlike for Social Anxiety Disorder). Interestingly, this implication of our theory is exactly what the DSM observes. Note that Panic Disorder is defined by unexpected panic attacks, the person does not necessarily have any idea what triggers them or any beliefs about why they happen, they just happen "out of the blue".

This does though raise the question, if someone has Panic Disorder, why don't they experience panic attacks all the time? The answer here is that just because panic attacks could happen all the time (which our theory suggests they could given the person's beliefs), they will only actually happen when the person for whatever reason starts to focus on the endogenous feelings and involuntary thoughts they have associated with panic attacks (and in doing so starts the feedback loop). Hence, we can explain the seeming randomness and variability of frequency of panic attacks in Panic Disorder, panic attacks just happen whenever the situation conspires to make the particular person concerned that panic attack might be possible. Here we can see how the person's beliefs about the trigger (factor 3) could be so important in shaping how frequently and when they occur. 
Overall then, the DSM diagnostic criteria for Panic Disorder also suggests the two necessary conditions would be fulfilled and the condition presents itself in a manner that is consistent with the three factors we theorised would influence it. From this we can see the only fundamental difference between Panic Disorder and Social Anxiety Disorder is the belief about how bad the fear is (very bad in case of Panic Disorder) and the type of belief about how endogenous feelings relate to the fear (i.e in Panic Disorder the fear is essentially the endogenous feelings themselves, rather than some external (context specific) consequence of them e.g embarrassment).

To highlight this lack of fundamental difference, it is interesting to think back to our description of how the feedback loop could explain the common experience of getting quite anxious before giving a presentation or public speaking (i.e an instance of social anxiety). If that description resonated with you then I hope that maybe we can start to see how a condition like Panic Disorder is really no different. Imagine that rather than believing the sweating and shaking might lead you to embarrass yourself in the presentation, you instead genuinely believed it might mean you were about to die or go insane. I think we can see it would make complete sense that you would get much more anxious much more rapidly, and as this rapid escalation of feelings happened, you can see you would start to think dying or going insane was becoming very likely, and then you might well just panic. Therefore, I believe we can start to see how even for anxiety conditions that appear very extreme (like Panic Disorder) could actually just be reflection of a mental process (the anxiety feedback loop) that most of us experience from time to time, just with a different set of beliefs about what our endogenous feelings mean.

Before we leave our discussion of Panic Disorder I also wish to highlight a special case of the anxiety feedback loop we have nearly touched on. In Panic Disorder we have discussed how the endogenous feelings are very closely related to the fear, i.e a very strong relationship. If we were to take this a step further we can see how we could have an anxiety feedback loop where the fear is itself the experience of the endogenous feelings and involuntary thoughts associated with an anxiety event i.e a fear of fear. Anxiety events are really not very pleasant and so it also seems entirely plausible that a person could see being really anxious as something to fear in itself. This possibility of a special case where the fear and the endogenous feelings are the same thing is one we will come back to in the next few discussions.

\section{3 | Specific Phobia ([3], p.197-202)}

The DSM diagnostic criteria for Specific Phobia describes it as "marked fear or anxiety about a specific object or situation", examples of the situations include "flying", "heights", and "animals". Crucially, the fear "is out of proportion to the actual danger posed by the specific object or situation", colloquially people often describe these then as 'irrational' fears.

The DSM definition of Specific Phobia appears to present an issue for the theory we have proposed. The diagnostic criteria does not really refer to the person having any belief about endogenous feelings being related to the fear. All it says is that the person experiences "out of proportion" anxiety when faced with some specific situation. This appears quite problematic for our theory as we can't establish that the first condition for the feedback loop holds for Specific Phobia. However, the DSM also doesn't present some other causal mechanism or set of beliefs at work that could cause the Specific Phobia and so it also doesn't invalidate this theory.

Therefore, I wish to propose a way our theory could explain how Specific Phobias come about and why they present themselves in the way the DSM describes. To illustrate this new perspective I would like to run through a hypothetical scenario.

Imagine the first time you get on a plane (often when you are young). Being fired into the air in a small metal tube strapped to two jet engines is not a particularly normal or relaxing experience.

Therefore, on this occasion, as you aren't sure what those funny noises are, turbulence is quite scary and you didn't think to study air safety statistics before bed time, you may well start to believe that these events suggest something very bad might be about to happen. This leads you completely rationally to become really very anxious. You are also likely to find the whole experience of getting very anxious in a small metal tube at 30,000 feet very unpleasant. You would probably get off the plane and think 'thank God that is over, that was very unpleasant'. 
You may well go away and talk to people or do some research and realise planes are extremely safe, turbulence is common and that those noises are completely normal. When you next go to fly someone may say, 'do you now see why flying isn't that dangerous?' and you will say 'yes it seems fine, I was just being silly'.

This seems like it should be the end of you getting very anxious on planes. The problem is you can't wipe your memory of how unpleasant your last flight was. Therefore, quite rationally, the next time you get on a plane you are very likely to think 'I really hope I don't feel like that again, it was very unpleasant'. In other words, you now actually don't fear flying but you do now have a fear of being very anxious on flights, i.e you now have a fear of fear. The problem is, as we discussed in Panic Disorder, the anxiety feedback loop is clearly entirely possible where the fear itself is the endogenous feelings and involuntary thoughts associated with anxiety. This thought then when you step on the plane will likely lead you to focus on whether you are experiencing the endogenous feelings of anxiety again, because you are on a plane again and you really don't want to get very anxious again, you may well notice you do have a low level of the endogenous feelings associated with anxiety. This will lead you to think 'god I hope those endogenous feelings don't mean I am going to get very anxious like last time' this focus will, as we know, lead the endogenous feelings to escalate, you will revise your probability of having an anxiety event (i.e the fear) and the feedback loop will start spiralling.

This feedback loop then will lead you to have an anxiety event that will feel and look basically like the first anxiety event. There is clearly a key difference though, you are no longer actually scared of flying because it is dangerous etc., you now are just scared of being scared whilst flying. If you don't understand this possibility though (due to the second necessary condition) then this slight of hand will look to both you and observers a lot like for some unknown reason you are now just irrationally scared of flying.

Once you have this mistaken secondary belief that you are irrationally scared of flying then every time you get on a flight you will likely be concerned that you will get excessively anxious. In other words, flights have become a trigger. Getting on a flight will lead you to focus on the endogenous feelings and involuntary thoughts associated with anxiety and in doing so trigger the feedback loop that causes the fear (anxiety) to come about.

This hypothetical scenario then starts to sounds quite a lot like how the DSM describes the characteristics of Specific Phobia. You can also basically replace flying in the scenario above with any other common phobia noted in the DSM (height, storms, spiders, dogs, medical procedures) and get a very similar plausible scenario. The DSM also explicitly notes how Specific Phobia can in many cases be traced back to a "traumatic event", "observation of" a traumatic event or a "panic attack in the to be feared situation" but also in many cases the person does not recall the specific reason as they were very young ("median age at onset is between 7 and 11 years"). In adults, the DSM notes Specific Phobia is "often as the result of experiences that are traumatic".

Therefore, the causal explanation we have proposed for how a phobia could develop using our theory appears to fit with the way the DSM describes the characteristics and development of Specific Phobia. It also has the benefit of explaining two of the more unusual observations of phobias.

First, phobias appear irrational. Phobias appear unusual because the sufferer usually knows that the plane is very unlikely to crash, or that the spider can't kill them, or that they aren't going to fall to their death standing behind a railing looking down. However, they seem still to get "out of proportion" anxiety in this very specific situation, yet they experience entirely normal levels of anxiety in lots of actually far more dangerous situations. From our explanation of how a phobia can develop we can see this is a mistake, the person isn't being 'irrational', they and any observers have simply misunderstood what the person now actually fears. The sufferer now, completely rationally, has a self fulling fear of fear itself, with the situation described by the phobia simply as the trigger.

Second, phobias are highly correlated with having other phobias. The DSM notes "the average individual with specific phobia fears three objects or situations, and approximately $75 \%$ of individuals with specific phobia fear more than one situation or object". This is also an usual presentation, why would having one seemingly irrational fear in one specific context be correlated with also having other irrational fears in other highly specific but completely different contexts. From our explanation we can now see why this correlation is actually what we would expect. If you are someone who really doesn't like the experience of getting anxious (i.e you have a fear of fear), you are quite likely to see triggers for the feedback loop in many situations that lead you to focus on the endogenous feelings and involuntary thoughts associated with anxiety. 
Overall, even though we can't explicitly tie our theory to the DSM diagnostic criteria for Specific Phobia, I believe we can provide a compelling account of how our theory could causally explain the development of a phobia and the unique characteristics of phobias. As we will see next, the account we gave above is also essentially what the DSM describes for another type of phobia.

\section{4 | Agoraphobia ([3], p.217-221)}

The DSM diagnostic criteria describes Agoraphobia as "marked fear or anxiety" in relation to two or more of "public transportation (e.g., automobiles, buses, trains, ships, planes)", "open spaces", "enclosed places", "standing in line or being in a crowd", "being outside of the home alone". Crucially, one of the criteria for a diagnosis of Agoraphobia is:

"The individual fears or avoids these situations because of thoughts that escape might be difficult or help might not be available in the event of developing panic-like symptoms or other incapacitating or embarrassing symptoms"

In other words, the DSM diagnostic criteria includes that the reason the person fears the situation is that they may experience endogenous feelings (i.e "panic-like symptoms or other incapacitating or embarrassing symptoms"). Therefore, it appears the DSM criteria of Agoraphobia explicitly fulfills the first necessary condition for the anxiety feedback loop, namely that the person believes a fear (being stuck or unable to get help when developing panic-like symptoms or other incapacitating or embarrassing symptoms) is related to endogenous feelings or involuntary thoughts (i.e panic-like symptoms or other incapacitating or embarrassing symptoms).

Even more interestingly, when we discussed Specific Phobia we noted there was no reference in the DSM to how/whether the fear was related to endogenous feelings or involuntary thoughts. This led us to present one plausible way it could be explained by our theory, in which we suggested we could see how a phobia was really the anxiety feedback loop based in a fear of fear, not the 'irrational' fear of some situation it is commonly characterised as. When we now look at Agoraphobia (note it has phobia in the name) the DSM is explicitly stating that for Agoraphobia we are often talking about a fear of fear ("in the event of developing panic-like symptoms").

Therefore, under our theory there appears to be significant overlap between Agoraphobia and Specific Phobia. In the case of Agoraphobia where the person holds the fear of "developing panic-like symptoms" (i.e a fear of fear) it seems we can use essentially the same causal explanation we gave for how a Specific Phobia could develop and present itself. Hence, I will not repeat this here.

However, the DSM definition of Agoraphobia is actually broader than for Specific Phobia. Where Specific Phobia just refers to feelings of "marked fear or anxiety", Agoraphobia refers to "developing panic-like symptoms or other incapacitating or embarrassing symptoms". Therefore, Agoraphobia is not necessarily a fear of fear. Plenty of other endogenous feelings could relate to the fear of "being stuck or unable to get help". Examples would include, the endogenous feelings associated with needing the toilet ("fear of incontinence"), the endogenous feelings associated with embarrassment (blushing, sweating, trembling etc.), the endogenous feelings associated with having a tight chest / trouble breathing (e.g hyperventilating) or endogenous feelings / involuntary thoughts the person (mistakenly) believes are associated with them losing control.

Overall though, in either case, we are talking about a fear that is directly related to endogenous feelings and so we can see that the DSM diagnostic criteria for Agoraphobia suggests the first necessary condition for the anxiety feedback loop would be fulfilled (and the second is given as discussed). Confined, open or crowded spaces also represent a common situation that would regularly trigger an anxiety event of this nature.

\section{5 | Generalised Anxiety Disorder ([3], p.222-226)}

I have left Generalised Anxiety Disorder to the end for a reason. The DSM's diagnostic criteria is very broad and, as we will discuss, somewhat at odds with the criteria I would expect for diagnosing an anxiety condition. This will lead us to define 
Generalised Anxiety Disorder slightly differently and provide a separate causal explanation which will be at odds with parts of the DSM's description.

The DSM describes Generalised Anxiety Disorder as "excessive anxiety and worry" about "a number of events or activities". Therefore, Generalised Anxiety Disorder does not really have a specific presentation or specific set of fears. In fact, given anxiety is completely normal (we live in a very uncertain world), Generalised Anxiety Disorder appears very hard to distinguish from normal anxiety.

The DSM proposes we can distinguish it via three criteria. First, the worry is "excessive". Second, the worry is more "pervasive". Third, the worry is more likely to be "accompanied by physical symptoms".

The problem is all three of these criteria are largely subjective, (how much is the 'correct' level of worry about passing exams to get a job?, how much 'should' you fear death or illness?, how often 'should' you worry about the decline of democracy?). Under the DSM it is the clinician who decides if the person is being 'normal' in the degree to which they worry about "a number of events or activities" or if the person has an anxiety disorder.

I find this rather troubling. As great as clinicians are I do not think they are in a good position to say what the 'correct' answers are to the deep philosophical questions about life and death on behalf of another person. Quite simply there is no objective baseline for what things we should worry about and to what degree they should concern us. Practically, even if there was a baseline, we can never observe someone else's actual level of anxiety (only their description), so it would be really very hard to compare individuals to the baseline to ever know if what they experience is actually "excessive" or "pervasive".

Therefore, I only really want to deal with a far narrower type of Generalised Anxiety Disorder, where the sufferer believes that their own level of worry is 'excessive' and the clinician agrees. This definition of Generalised Anxiety Disorder has the benefit of being clearly distinguishable from normal anxiety. When humans get 'normally' anxious they usually don't find their own anxiety response odd, they know why they have that anxiety response because they know they are anxious about some fear. In contrast, where the sufferer themselves finds their own level of anxiety unusual and can see it is out of line with their own beliefs about how bad the fear is then this appears quite different. This also matches how we defined anxiety as a separate condition in the section 'Anxiety Conditions'.

On this narrower definition of Generalised Anxiety Disorder we have actually already discussed how this can occur. When we discussed Specific Phobia we highlighted the possibility of a fear of fear. In the case of Specific Phobia this was combined with an (incorrect) secondary belief that the cause/trigger of the anxiety feedback loop was some situation (hence why phobias are so context specific). However, we noted how under our theory, the anxiety feedback loop based in a fear of fear could actually happen at anytime assuming the person believed it possible and focused on their endogenous feelings and involuntary thoughts.

Therefore, we can see how Generalised Anxiety Disorder could actually be the same as Specific Phobia except the person does not have a mistaken belief that they need to be in some specific situation. Instead, whenever the sufferer gets 'normally' anxious about some life event, they start to notice the endogenous feelings and involuntary thoughts associated with anxiety. If they are someone who really doesn't like the experience of excessive anxiety (i.e they have a fear of fear), then observing these anxious feelings could trigger the anxiety feedback loop that escalates the level of anxiety they experience beyond what appears appropriate for the situation and towards the level of anxiety they fear.

Once again then we can see that maybe the person is not being excessive, irrational or abnormal but rather they genuinely really dislike the experience of getting anxious, and this fear of fear, under this theory, is self-fulling (whilst the two necessary conditions are in place).

Our theory then cannot explain the DSM diagnostic criteria for Generalised Anxiety Disorder. It can though explain an alternative, narrower, condition that would present itself in a very similar way to the DSM description of Generalised Anxiety Disorder but would exclude those instances where the sufferer themselves does not believe their anxiety is 'excessive'. 


\section{6 | Other Conditions}

This completes our discussion of the specific anxiety conditions as defined in the DSM. However, as our theory clearly rests on different foundations to the DSM, there is evidently the possibility our theory can provide insight into other anxiety conditions.

From the perspective of this theory, the DSM actually contains a relatively short list of the most common or extreme examples of the anxiety feedback loop. The theory we have proposed suggests these instances are just some notable examples on a broad spectrum of possible ways people could experience this feedback loop in their consciousness. There is likely to be numerous other milder or less common situations involving beliefs about how endogenous feelings could be related to something the person fears where the same anxiety feedback loop could also exist.

Furthermore, splitting up anxiety conditions into discrete categories based on presentation (Panic Disorder) or situation (Social Anxiety Disorder, Agoraphobia etc.) also makes little sense from the perspective of our theory. If the same general feedback loop is operating across conditions then it would make more sense to discuss anxiety conditions in terms of the three factors we discussed in the section 'Factors Affecting the Anxiety Feedback Loop' which determine the specific characteristics of the feedback loop.

\section{7 | Summary}

From this discussion of the DSM diagnostic criteria for anxiety conditions, I think we can see that the necessary conditions for the anxiety feedback loop are either directly specified in the DSM or that we can construct a plausible casual explanation for the characteristics described in the DSM. This suggests our theory may be well placed as a possible description of reality. The exception to this is Generalised Anxiety Disorder for the reasons we have discussed.

I believe it is more than a coincidence that, despite the infinitely many possible fears humans can have, the DSM repeatedly refers to fears that are directly or easily related to the person's endogenous feelings and that it so happens it is only these types of fear that could generate the anxiety feedback loop we have theorised should exist.

What's more, from the fairly basic observations of our own conscious experience we made in the section 'A Model of My Consciousness', we can see the potential for a feedback loop between endogenous feelings and deliberate thoughts. It is then interesting that this theoretical feedback loop would be expected to present itself in a way that is typical of the characteristics described in the DSM for anxiety conditions. Furthermore, the factors that we theorised would determine the feedback loop's specific characteristics also appear to match the presentation of different anxiety conditions observed in reality.

Overall, I hope this discussion of the DSM goes someway to substantiating this theory and also demonstrates how it can be applied in practice.

\section{7 | A THEORY OF ALLEVIATING ANXIETY CONDITIONS}

If we were to subscribe to this theory, it evidently has implications for how we can treat anxiety conditions and why therapeutic treatments are effective. I would suggest the theory gives rise to two therapeutic approaches to alleviating anxiety.

\section{1 | The Direct Approach}

We identified two necessary conditions for the anxiety feedback loop to occur, (1) the person must be in a situation where they believe their endogenous feelings are meaningfully related to the probability of some fear occurring, (2) the person must not 
fully understand the dynamics of this feedback loop in their consciousness. The direct approach to alleviating anxiety would be to try and break the feedback loop by removing one or both of these necessary conditions.

The first step under the direct approach would be to discuss and identify the person's beliefs about these two necessary conditions, i.e what specific beliefs does the person hold that means these conditions are fulfilled.

The question would then become whether it is possible to change the person's beliefs such that one (or both) of these necessary conditions is no longer fulfilled. Which necessary condition to focus on and how this can be achieved is not that simple and so I will discuss each necessary condition separately.

\subsection{1 | Removing the first necessary condition}

The ultimate goal of this therapy would be to help the person realise that their endogenous feelings are in fact not meaningfully related to the probability of the fear. This would break the feedback loop as the person would no longer have a reason to focus on the endogenous feeling or the fact it is escalating.

This could be a fruitful approach if the person's beliefs about the relationship between the endogenous feelings and the fear are clearly mistaken. For example, imagine someone who believed their heart racing, sweating, shaking and so on (endogenous feelings) meant that they were about to lose control of their actions and might do something terrible (the fear). We can see how by discussing with the person that these feelings are very common in people and that they have no ability to cause us to take actions against our will, we may be able to start to change the person's belief that these endogenous feelings are related to the fear. If over time the person comes to accept that the endogenous feelings are not related to the fear, we can see this would break the feedback loop. Now when the person initially experiences the endogenous feelings, they will no longer believe they are particularly important as they are not related to a fear, they will not focus their attention on the feelings, the feelings will no longer escalate and neither will the associated level of anxiety.

However, the issue is that for a large proportion of anxiety conditions these beliefs are not mistaken, they are often entirely correct. This would include types of anxiety (e.g social anxiety) that involve the fear of embarrassment due to the symptoms of anxiety or anxiety that involves the fear of fear itself (e.g phobias). In these cases, whilst the second necessary condition is still in place, the person's belief that the endogenous feelings are related to the fear is true and often a self fulling prophecy.

Therefore, if mistaken beliefs are involved, a therapeutic approach that tries to remove the first necessary condition may be both the easiest and most effective method for alleviating anxiety. In many cases though, this approach is not possible because the person's beliefs that fulfill the first necessary condition are correct.

It is worth noting how a therapeutic approach that tried to challenge someone's mistaken belief about their endogenous feelings being related to the fear would often in practice look very similar to exposure therapy. We would often want to encourage the person to put themselves in the situation where they believe the endogenous feelings are related to the fear (i.e expose themselves to it) in order to show them that they are incorrect about the relationship between endogenous feelings and the fear.

\subsubsection{Removing the second necessary condition}

Before we begin this part, I would like to note that the following description may well seem quite odd and unintuitive. It took me many months of introspection to understand what options we have when faced with the perverse dynamics of the anxiety feedback loop.

The ultimate goal of a therapy targeting this second necessary condition would be to help the person realise that by their very nature the endogenous feelings involved will escalate if they focus their deliberate thoughts on them (i.e the dynamics of the anxiety feedback loop). If the person observes this in themselves and develops this belief, then they will rationally decide to no longer focus their deliberate thoughts on the endogenous feeling. Without focus, the feedback loop is broken and the endogenous feelings will cease to escalate. 
Even when I write this now, the idea the person could/would choose not to focus on the endogenous feelings seems wrong. This though is for good reason, the theory we have proposed results in a deeply unintuitive conclusion. Therefore, to illustrate why the previous paragraph makes sense, it is worth going over how I came to it.

I could see the anxiety feedback loop playing out in my head and I could see the perverse logic that by deliberately thinking about the endogenous feelings I was actually making the thing I feared seemingly more likely. Yet the question that I repeatedly struggled to answer is as follows: if I believe my endogenous feelings escalating provides information about the fear, then doesn't this mean I 'should' focus on the fact they are escalating because it is telling me something useful about how likely the fear is and when the fear might happen?

This statement sounds very true, if there is new information (e.g changes in endogenous feelings) that tells me about the likelihood of something I fear, then surely I 'should' think about that new information and what it might mean. It sounds so true because in almost all other situations in life it is so true. When out of the corner of my eye I notice something flying towards my head, it is a very good idea to focus on it. When I hear rustling in the vegetation whilst trekking in the rainforest, it is probably clever to think about it and what it could be. When I am driving in my car and don't want to crash, it makes a lot of sense to focus on the movements of the cars around me. Therefore, it appears we are predisposed to believe the rule: We 'should' focus on things we believe are associated with the events we fear. This belief is generally an extremely successful and applicable approach to avoiding risks in a very uncertain world.

I came to realise though endogenous feelings are one rare but crucial exception to this rule. If the act of using your deliberate thoughts to think about something (e.g focusing on the endogenous feelings) could itself be the very thing generating the 'new information' (e.g changes in our conscious experience of endogenous feelings) and if there is no other external way to verify the 'new information' (e.g only we can observe our consciousness), then this rule appears to fall apart. It falls apart because what appears to be 'new information' (changes in endogenous feelings) could always actually just be a reflection of the fact you decided to focus on it and believe it is important, and so there isn't actually any meaningful 'new' information to gain. If there is no useful information to gain by focusing on the endogenous feeling, then there is no longer a reason why we 'should' do so.

This is precisely the situation we find ourselves in with the anxiety feedback loop. When we decide to focus on our endogenous feelings and in turn observe them escalating, it is quite simply a mistake to interpret this as 'new information', all it is telling you is that you decided to focus on them, and you already knew that. Therefore, in this situation we appear to only have two options, (1) focus on the endogenous feeling, now in the full knowledge this will likely lead us to experience them escalating, (2) don't focus on the endogenous feeling, and our conscious experience of them may or may not change. Option (1) then both provides no new information (as the escalation is entirely predictable) and also appears to (and in some cases actually does) make the situation worse as it causes your endogenous feelings to escalate. In contrast, option (2) does not cause the endogenous feelings you fear to escalate and also still leaves open the possibility for new information. The normal rule then is completely reversed, in this rare situation, not focusing on the thing you associate with the fear becomes the best thing to do. Crucially, this is true whether our beliefs about the relationship between the endogenous feeling and the fear (i.e the first necessary condition) are correct or completely mistaken.

Overall then, the essence of a therapy targeting the second necessary condition is that by understanding our consciousness in more detail, we start to see (and gradually believe) that changes in our endogenous feelings might not be telling us what we previously thought. We start to realise that maybe the only control we have is to try and let go of the involuntary thoughts bringing the endogenous feelings to our attention and to try to focus our deliberate thoughts on something else. Over time you come to understand that what really seemed like something you 'should' think about and even something you 'should' be able to control can actually only ever be made worse by focusing on it. Choosing to 'ignore' something you believe is associated with a fear is initially a very difficult and weirdly scary experience. However, through practice and understanding, you come to observe the truth in this way of viewing our consciousness, and you will gradually build a belief in a different rule for dealing with your endogenous feelings. Then when you are in situations where the anxiety feedback loop is possible you will quite simply not see the point in focusing on your endogenous feelings because you will believe that no good nor useful information can come of it, and thus break the feedback loop.

This therapeutic approach is by far the most difficult (both for the person and therapist) and is focused on educating people about the nature of their own conscious experience. However, if this theory is correct, this approach would also be the most 
effective as it does not alleviate just one instance of anxiety about a particular fear but instead structurally changes the person's relationship with their endogenous feelings and so alleviates the possibility of the anxiety feedback loop in general. Hence this approach can be applied to any form of anxiety resulting from the anxiety feedback loop. This is why I started the theory emphasising the importance of the possibility that people may well hold in-accurate beliefs about their consciousness and how we currently lack any formal education or discussion of the subject. If this therapeutic approach works, it shouldn't really be called therapy, it should be called mental education and provided to everyone.

It is also worth noting how this therapeutic approach would actually result in outcomes that look very similar to current therapeutic techniques such as mindfulness, breathing exercises, meditation etc. All these current practical steps for dealing with anxiety can be seen to be fundamentally saying 'even though it doesn't seem right, the best way to deal with anxiety events is to think about something else'. This theory provides us and the sufferer with the explanation why.

\subsection{3 | Summary}

The direct approach to alleviating anxiety works by trying to remove one of the two necessary conditions that are required for the anxiety feedback loop to occur. Changing the person's belief about the endogenous feeling being related to the fear is by far the easiest, but it relies on the person's beliefs being wrong, which is often not the case. In contrast, changing the person's beliefs about how the anxiety feedback loop works and particularly the endogenous nature of the feelings involved is much harder, but can be applied to all anxiety conditions.

Under this theory, the direct approach is the only way to prevent the anxiety feedback loop and anxiety events. This is because it represents the only way to avoid the conditions in which the anxiety feedback loop can occur.

However, in many cases we can go a long way to alleviating the intensity of anxiety events without changing these core beliefs directly. It is to this indirect approach which we now turn to.

\section{2 | The Indirect Approach}

Assuming the two necessary conditions for the anxiety feedback loop are in place, we identified three general factors which determine the characteristics of how the feedback loop plays out. The indirect therapeutic approach to alleviating anxiety would be to try and modify these three factors and in doing so reduce the intensity or frequency of anxiety events. By taking each factor in turn we can see how this could be possible in practice.

\subsection{1 | Factor 1 - The belief about how closely endogenous feelings are related to the fear}

When we introduced this factor we discussed how if the person believes endogenous feelings are the only factor that cause (or are all the possible evidence of) the fear then they will experience more intense anxiety events. In contrast, if the person believes endogenous feelings are only one of many factors (or one of many pieces of evidence for) the fear then they will experience relatively less intense anxiety events.

Therefore, the goal of therapy targeting this factor would be to encourage the person to see that the endogenous feeling is not as closely related to the fear as they currently believe. In doing so the person would lower their own estimate of how likely the fear is based on observing their escalating endogenous feelings. This in turn will reduce how anxious the person becomes when they experience the anxiety feedback loop.

To give an example of how this could work, let's consider a case of health anxiety. Imagine a person believed that their heart racing and a feeling of tightness in their chest (endogenous feelings) is evidence they are likely about to die from a heart attack (the fear). In this case the belief that the endogenous feelings are related to the fear (first necessary condition), is not definitively incorrect, these symptoms may precede some cases of heart attacks. However, what we can see is that the person's belief about 
how close the relationship is, is incorrect, i.e these endogenous feelings do not mean you are likely to have a heart attack. If we were to discuss with them how these symptoms are far more common in anxiety itself and that a whole host of other symptoms would be required before you are rushed to hospital, then we may be able to change their belief about how closely related these feelings are to the fear. In doing so we would reduce the intensity of the person's anxiety events as in future they will no longer see the escalating endogenous feelings as significant evidence they are likely to have a heart attack, instead they will only see them as one small piece of evidence, which may or may not mean they are going to have a heart attack.

Once again though this therapeutic approach will only be effective if the person's belief about how close the relationship is, is incorrect. In many cases of anxiety, this is not the case. Hence the possibility of this therapeutic method will depend on the specific instance.

\subsection{2 | Factor 2 - Beliefs about how bad the fear would be}

A therapeutic approach targeting this factor I think would be very recognisable. The goal would be to try and lead the person to change their perspective of the fear and in doing so downgrade how bad they believe the fear happening would be. If the person lowers their belief about how bad the fear would be then the person will become less anxious as they observe their endogenous feelings escalate.

Clearly the effectiveness of this therapeutic approach will also be heavily reliant on how realistic the person's evaluation of the fear is initially. It is very hard to convince someone that dying wouldn't be that bad. Much easier if you are trying to convince them that sweating or blushing in a social situation wouldn't be as noticeable or embarrassing as they currently imagine.

\subsubsection{Factor 3 - The belief about the trigger}

I don't think there is really a general therapeutic approach using this factor. However, there is the possibility that in some instances we may be able to reduce the number of situations in which the person believes the endogenous feelings are related to the fear.

For example, in a case of agoraphobia, by discussing ways in which the person could either get out of the situation or manage the endogenous feelings, we may be able to reduce the number of places the person considers 'confined' and in doing so reduce the number of triggers. This in turn would lower the frequency of anxiety events in their everyday lives.

At this point it would be remiss of me not to highlight that discussing this factor could in some instances result in the person's anxiety condition becoming more generalised. We have described on a number of occasions how the person's belief about what triggers their anxiety may be mistaken. In the case of phobias, this mistaken belief can actually be beneficial as it leads the person to believe that their fear of fear can only occur in a specific set of situations. In reality, this theory suggests a fear of fear itself could actually happen in any situation, all it takes is for the person to think it possible and focus on the endogenous feelings associated with anxiety. Therefore, in these cases, when leading someone to a more accurate understanding of what causes their anxiety we may in the short term cause them to experience anxiety events in new situations that they previously had not considered could be triggers. However, if we wish to alleviate these forms of anxiety, this new understanding is a key foundation for the direct method of therapy and often the only approach available.

\subsection{4 । Summary}

The indirect therapeutic approach to alleviating anxiety works by adapting the person's beliefs so they view the anxiety feedback loop as less important. This could be because they now believe the fear is less bad, they see the endogenous feelings as less connected to the fear or they see fewer situations in which the fear can occur. Hence the indirect approach can be a powerful way to reduce the intensity of the person's anxiety events, and in some cases this may be enough for the person to no longer consider their anxiety an issue in itself. 
However, none of the indirect approaches actually breaks the anxiety feedback loop and they all are only really possible if the person's beliefs are mistaken in some demonstrable way. Therefore, a combination of the indirect approach with the direct approach may well be required.

Overall, the proposed theory gives rise to a number of possible therapeutic approaches depending on which aspect of the anxiety feedback loop we wish to target. We have also noted how these approaches would in practice encompass / explain existing therapeutic techniques such as exposure therapy, mindfulness and coping strategies. The possibility and efficacy of the approaches will depend on the characteristics of the individual case. However, our theory suggests the only approach which is applicable to all anxiety conditions is to educate the person on the nature of their consciousness and the anxiety feedback loop itself and in doing so remove the second necessary condition for the feedback loop to exist.

\section{8 | CONCLUSION}

We started by identifying the different components of our conscious experience and how they related to one another. From this we saw that the system described by these components would have the possibility for feedback loops. We then identified a particular feedback loop between endogenous feelings and deliberate thoughts that would be characterised by an escalating process as thoughts beget feelings and vice versa. By combining this feedback loop with the idea that the person may believe that the endogenous feelings involved could be related to something they fear, we saw that such a feedback loop would generate many of the general characteristics we observe in anxiety events. Studying this anxiety feedback loop in more detail we identified two necessary conditions that must be in place for the feedback loop to occur, explaining the context specific nature of anxiety conditions. We also identified three general factors that would shape how a particular instance of the anxiety feedback loop would present itself.

We then applied this theory to show that the anxiety feedback loop and the factors affecting it could be used to explain the clinical anxiety conditions as described in the DSM. Finally, we outlined how the theory leads to a direct and indirect set of therapeutic approaches, which would share many techniques with existing therapies. Crucially though, we identified one new therapeutic approach that could be applied in all cases, namely, education and discussion about the components and dynamics of our own conscious mind, specifically the existence of the anxiety feedback loop.

Ultimately, everything we have discussed flows directly from some fairly trivial observations of our own conscious experience. I believe the fact this theory can seemingly explain so much without needing to resort to dysfunction, irrationality, biology or abstract theories of mind is notable in itself. More importantly, this means the theory can be observed and tested by everyone of us because it speaks directly about what we can observe in ourselves. I believe this theory is true of me and my anxiety. I hope it can help you understand your own or other peoples'.

In the same way we look back and view not educating people on sexual health as a grave oversight, I believe we will look back and view the fact we rarely educate people about what to expect from their consciousness, what the different components are, what the components mean, what control we have over them and what dynamics they create, as a significant failing. This theory suggests an anxiety condition does not indicate there is something 'wrong' with the person, it indicates the person misunderstands themselves, and this misunderstanding leads them to entirely rationally think and do things that lead to very negative states of mind.

This theory of anxiety is also only one thread that drops down from conceptual model of our conscious experience we discussed in the section 'A Model of My Consciousness'. I believe viewing our conscious experience this way opens up a range of practical and philosophical questions that I have not been able to address here but which could have significant implications for how we view ourselves. One avenue for how this theory could be extended to possibly understand Intrusive Thoughts and OCD is laid out in the postscript to this essay. 


\section{9 | POSTSCRIPT - TOWARDS A THEORY OF INTRUSIVE THOUGHTS AND OCD}

I wish to suggest that from what we have discussed in our theory of anxiety conditions we can also see the beginnings of a theory of Intrusive Thoughts and OCD based in a different feedback loop but which has essentially the same dynamics.

\subsection{1 | The Involuntary Thought - Deliberate Thought Feedback Loop}

When we discussed the components of my consciousness I also noted that we experience having thoughts which we do not directly control and seem to just 'pop into our head', i.e involuntary thoughts. Crucially, we noted how the thoughts that 'pop into our head' are often related to what we are deliberately thinking about at the time and what we believe is important.

If we accept then that we have thoughts we do not directly control, the contents of which are often related to what we are deliberately thinking about / believe is important, and if we hold beliefs that the content of these involuntary thoughts is important (i.e maybe we believe it reflects our deepest desires, maybe we believe it is telling us that something bad is going to happen, or maybe we believe it is just very unpleasant), then we can start to see there is the possibility of a different feedback loop in our consciousness.

We experience an involuntary thought, our beliefs lead us to think this thought is important, so we focus our deliberate thoughts on what the involuntary thought might mean or how we can get rid of it, this deliberate thinking leads more related involuntary thoughts to pop into our head, these further thoughts lead us to deliberately think even more about why these involuntary thoughts are occurring or what they are telling us, and so on.

\subsection{2 | The Feedback Loop as it Relates to OCD / Intrusive Thoughts}

If we add that the reason the person believes the involuntary thoughts are important is that they believe they are related to something they fear, then this new feedback loop would appear to describe many of the general characteristics of the conscious experience of Intrusive Thoughts / OCD. This feedback loop would be characterised by having a trigger, after which the person increasingly focuses on the meaning or contents of their involuntary thoughts, thus triggering further or more severe involuntary thoughts, leading them to become increasingly disturbed / anxious about why the involuntary thoughts are occurring and the possibility of the fear.

If we then copy pasted 'involuntary thoughts' for 'endogenous feelings', and 'escalating endogenous feelings' for 'additional or more severe involuntary thoughts', the rest of the theory appears largely unchanged. The feedback loop would have essentially the same two necessary conditions for it to occur and largely the same factors that would influence its characteristics.

\subsection{3 | Explaining the DSM}

Similarly, if we look at the DSM we can see the necessary conditions for this Intrusive Thought / OCD feedback loop would be fulfilled by the diagnostic criteria and the feedback loop would largely explain the presentation described.

The DSM describes how the sufferer experiences "obsessions" involving "persistent thoughts", "images (e.g., of violent or horrific scenes)" or "urges (e.g., to stab someone)", crucially these thoughts are "intrusive and unwanted" (i.e involuntary). The person's compulsions are to "reduce the distress triggered by obsessions or to prevent a feared event".

In other words, the person believes their involuntary thoughts are related to something they fear (the fear may be simply the unpleasantness of the thought itself, i.e "the distress", or what the thought 'means' about themselves/the world, i.e "prevent a feared event"). This belief leads the sufferer to see their involuntary thoughts as important and as such when they arise the 
person believes they should take actions or deliberately think things to assuage them or to reduce the risk of the fear. This leads to further and/or more severe involuntary thoughts to arise and so on.

From this we can see the first condition of the anxiety feedback loop is fulfilled, the second condition is assumed to be fulfilled by definition as the DSM does not mention the cause being a feedback loop. This means the feedback loop could occur in these situations. Furthermore, we can see the feedback loop would fit with the DSM's description of the characteristics of OCD / Intrusive Thoughts.

\subsubsection{A Alleviation and Summary}

As the necessary conditions and factors remain largely unchanged, it seems a very similar set of therapeutic approaches would arise to those in our theory of anxiety. This should be unsurprising given we currently often use the same existing therapies to treat both sets of conditions.

Overall then, whilst this is clearly in no way a complete theory, it does appear to be a possibly fruitful approach to understanding what causes OCD / Intrusive Thoughts and how we might be able to alleviate them.

\section{0 | REFERENCES}

1. Clark, D. A., \& Beck, A. T. (2011). Cognitive therapy of anxiety disorders: Science and practice. Guilford Press.

2. Ellis, A., \& MacLaren, C. (1998). Rational emotive behavior therapy: A therapist's guide. Impact Publishers.

3. American Psychiatric Association, \& American Psychiatric Association. (2013). Diagnostic and statistical manual of mental disorders: DSM-5. United States.

\section{1 | AUTHOR PUBLIC KEY}

Generated using: openssl ecparam -genkey -name secp384r1

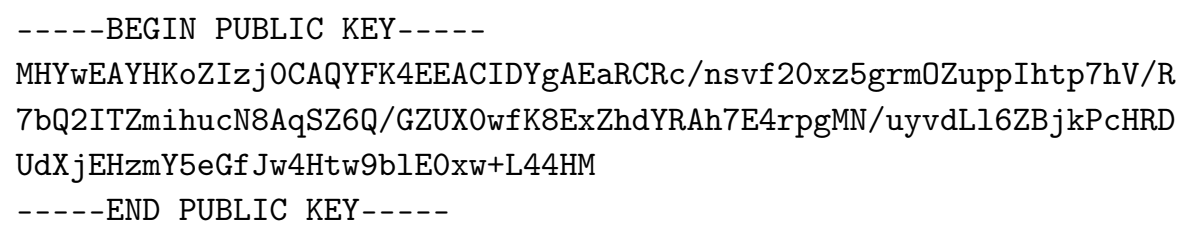

